\title{
Probing eV-scale axions with CAST
}

E. Arik ${ }^{1, \dagger}$, S. Aune ${ }^{2}$, D. Autiero ${ }^{3,18}$, K. Barth ${ }^{3}$, A. Belov ${ }^{4}$, B. Beltrán ${ }^{5,19}$, S. Borghi ${ }^{3,20}$, G. Bourlis ${ }^{21}$, F. S. Boydag ${ }^{1, \dagger}$, H. Bräuninger ${ }^{6}$, J. M. Carmona ${ }^{5}$, S. Cebrián ${ }^{5}$, S. A. Cetin ${ }^{1}$, J. I. Collar ${ }^{7}$, T. Dafni ${ }^{5}$, M. Davenport ${ }^{3}$, L. Di Lella ${ }^{3,22}$, O. B. Dogan ${ }^{1, \dagger}$, C. Eleftheriadis ${ }^{8}$, N. Elias $^{3}$, G. Fanourakis ${ }^{9}$, E. Ferrer-Ribas ${ }^{2}$, H. Fischer ${ }^{10}$, P. Friedrich ${ }^{6}$, J. Franz ${ }^{10}$, J. Galán ${ }^{5}$, T. Geralis ${ }^{9}$, I. Giomataris ${ }^{2}$, S. Gninenko ${ }^{4}$, H. Gómez ${ }^{5}$, R. Hartmann ${ }^{6,23}$, M. Hasinoff ${ }^{11}$, F. H. Heinsius ${ }^{10,24}$, I. Hikmet ${ }^{1, \dagger}$, D. H. H. Hoffmann ${ }^{12}$, I. G. Irastorza ${ }^{5}$, J. Jacoby ${ }^{13}$, K. Jakovčić ${ }^{14}$, D. Kang ${ }^{10,25}$, K. Königsmann ${ }^{10}$, R. Kotthaus ${ }^{15}$, M. Krčmar ${ }^{14}$, K. Kousouris ${ }^{9,26}$, M. Kuster ${ }^{6,12}$, B. Lakić ${ }^{14}$, C. Lasseur ${ }^{3}$, A. Liolios ${ }^{8}$, A. Ljubičić ${ }^{14}$, G. Lutz ${ }^{14}$, G. Luzón ${ }^{5}$, D. Miller ${ }^{7}$, J. Morales ${ }^{5}$, T. Niinikoski ${ }^{3}$, A. Nordt ${ }^{6,12}$, A. Ortiz ${ }^{5}$, T. Papaevangelou ${ }^{2}$, M. J. Pivovaroff ${ }^{16}$, A. Placci ${ }^{3}$, G. Raffelt ${ }^{15}$, H. Riege ${ }^{3,12}$, A. Rodríguez ${ }^{5}$, J. Ruz ${ }^{5}$, I. Savvidis ${ }^{8}$, Y. Semertzidis ${ }^{17,27}$, P. Serpico ${ }^{15,28}$, R. Soufli ${ }^{16}$, L. Stewart ${ }^{3}$, K. van Bibber ${ }^{16}$, J. Villar ${ }^{5}$, J. Vogel ${ }^{10}$, L. Walckiers ${ }^{3}$, K. Zioutas ${ }^{17,3}$ (CAST Collaboration)

${ }^{1}$ Dogus University, Istanbul, Turkey

${ }^{2}$ CEA, IRFU, Centre de Saclay, Gif-sur-Yvette, France

${ }^{3}$ European Organization for Nuclear Research (CERN), Genève, Switzerland

${ }^{4}$ Institute for Nuclear Research (INR), Russian Academy of Sciences, Moscow, Russia

${ }^{5}$ Instituto de Física Nuclear y Altas Energías, Universidad de Zaragoza, Zaragoza, Spain

${ }^{6}$ Max-Planck-Institut für extraterrestrische Physik, Garching, Germany

${ }^{7}$ Enrico Fermi Institute and KICP, University of Chicago, Chicago, IL, USA

${ }^{8}$ Aristotle University of Thessaloniki, Thessaloniki, Greece

${ }^{9}$ National Center for Scientific Research "Demokritos", Athens, Greece

${ }^{10}$ Albert-Ludwigs-Universität Freiburg, Freiburg, Germany

${ }^{11}$ Department of Physics and Astronomy, University of British Columbia, Vancouver, Canada

12 Technische Universität Darmstadt, IKP, Darmstadt, Germany

13 Johann Wolfgang Goethe-Universität, Institut für Angewandte Physik, Frankfurt am Main, Germany

14 Rudjer Bošković Institute, Zagreb, Croatia

15 Max-Planck-Institut für Physik, München, Germany

${ }^{16}$ Lawrence Livermore National Laboratory, Livermore, CA, USA

17 Physics Department, University of Patras, Patras, Greece 
18 Present address: Institut de Physique Nucléaire, Lyon, France

19 Present address: Department of Physics, University of Alberta, Edmonton, Alberta, Canada

${ }^{20}$ Present address: Department of Physics and Astronomy, University of Glasgow, Glasgow, UK

${ }^{21}$ Present address: Hellenic Open University, Patras, Greece

${ }^{22}$ Present address: Scuola Normale Superiore, Pisa, Italy

${ }^{23}$ Present address: PNSensor GmbH, Rmerstrasse 28, München, Germany

${ }^{24}$ Present address: Ruhr-Universität Bochum, Bochum, Germany

${ }^{25}$ Present address: Institut für Experimentelle Kernphysik, Universität Karlsruhe, Karlsruhe, Germany

${ }^{26}$ Present address: Fermi National Accelerator Laboratory, Batavia, Illinois, USA

${ }^{27}$ Present address: Brookhaven National Laboratory, Upton, New York, USA

${ }^{28}$ Present address: European Organization for Nuclear Research (CERN), Genève, Switzerland

$\dagger$ Deceased

E-mail: eferrer@cea.fr

\section{Abstract.}

We have searched for solar axions or other pseudoscalar particles that couple to two photons by using the CERN Axion Solar Telescope (CAST) setup. Whereas we previously have reported results from CAST with evacuated magnet bores (Phase I), setting limits on lower mass axions, here we report results from CAST where the magnet bores were filled with ${ }^{4} \mathrm{He}$ gas (Phase II) of variable pressure. The introduction of gas generates a refractive photon mass $m_{\gamma}$, thereby achieving the maximum possible conversion rate for those axion masses $m_{\mathrm{a}}$ that match $m_{\gamma}$. With 160 different pressure settings we have scanned $m_{\mathrm{a}}$ up to about $0.4 \mathrm{eV}$, taking approximately $2 \mathrm{~h}$ of data for each setting. From the absence of excess X-rays when the magnet was pointing to the Sun, we set a typical upper limit on the axion-photon coupling of $g_{\mathrm{a} \gamma} \lesssim 2.2 \times 10^{-10} \mathrm{GeV}^{-1}$ at $95 \%$ CL for $m_{\mathrm{a}} \lesssim 0.4 \mathrm{eV}$, the exact result depending on the pressure setting. The excluded parameter range covers realistic axion models with a Peccei-Quinn scale in the neighborhood of $f_{\mathrm{a}} \sim 10^{7} \mathrm{GeV}$. Currently in the second part of CAST Phase II, we are searching for axions with masses up to about $1.2 \mathrm{eV}$ using ${ }^{3} \mathrm{He}$ as a buffer gas.

PACS numbers: 95.35.+d; 14.80.Mz; 07.85.Nc; 84.71.Ba 


\section{Introduction}

The most promising experimental approach to search for axions or other pseudoscalar particles is to use their two-photon interaction that is traditionally written in the form

$$
\mathcal{L}_{\mathrm{a} \gamma}=-\frac{1}{4} g_{\mathrm{a} \gamma} F_{\mu \nu} \tilde{F}^{\mu \nu} a=g_{\mathrm{a} \gamma} \mathbf{E} \cdot \mathbf{B} a,
$$

where $a$ is the axion field, $F$ the electromagnetic field-strength tensor, $\tilde{F}$ its dual, $\mathbf{E}$ the electric, $\mathbf{B}$ the magnetic field, and $g_{\mathrm{a} \gamma}$ the axion-photon coupling constant. This interaction implies the conversion $a \leftrightarrow \gamma$ in the presence of external electric and magnetic fields. One strategy (see [1] for a review) to search for this effect is Sikivie's helioscope technique where a long dipole magnet is oriented towards the Sun [2]. Axions with energies of a few $\mathrm{keV}$ would be produced in the hot solar interior by the transformation of thermal photons into axions in the electric fields of the charged particles of the solar plasma, the Primakoff effect [3,4], and re-converted into x-rays within the dipole magnet. This conversion in a macroscopic $B$ field is best viewed as a particle oscillation phenomenon in analogy to neutrino flavor oscillations [5]. Accordingly, the conversion probability in a $B$-field region of length $L$ is [6]

$$
P_{\mathrm{a} \rightarrow \gamma}=\left(\frac{g_{\mathrm{a} \gamma} B}{q}\right)^{2} \sin ^{2}\left(\frac{q L}{2}\right)
$$

where in vacuum $q=m_{\mathrm{a}}^{2} / 2 E$ is the photon-axion momentum difference (we use natural units with $\hbar=c=1$ ).

An early helioscope experiment was performed in Brookhaven in the early 1990s [7] and later a much more sensitive search in Tokyo [8-10]. The largest and most sensitive helioscope, the CERN Axion Solar Telescope (CAST), has taken data since 2003 and has provided the most restrictive constraints on the axion-photon coupling $[11,12]$, superseding well-known astrophysical limits [13]. The limit, $g_{\mathrm{a} \gamma} \lesssim 8.8 \times 10^{-11} \mathrm{GeV}^{-1}$, obtained in the first phase of CAST (CAST-I)[12] applies only for masses $m_{\mathrm{a}} \lesssim 0.02 \mathrm{eV}$. This is seen most easily by rewriting the conversion probability in the form

$$
P_{\mathrm{a} \rightarrow \gamma}=\left(\frac{g_{\mathrm{a} \gamma} B L}{2}\right)^{2} \frac{\sin ^{2}(x)}{x^{2}},
$$

where $x \equiv q L / 2$. The degradation of sensitivity to $g_{\text {a } ~}$ when going to high masses follows then from the $x^{-2}$ suppression of the conversion probability when $x \gg 1$. For the magnet length $L \simeq 9.26 \mathrm{~m}$ and the typical $4 \mathrm{keV}$ energy of solar axions, the sensitivity declines when $x \gtrsim 1$, or $m_{\mathrm{a}} \gtrsim \sqrt{4 E / L} \simeq 0.02 \mathrm{eV}$.

It has long been recognized that the sensitivity of axion helioscope experiments can be extended to larger masses if one fills the conversion region with a suitable buffer gas, providing the photons with an effective mass $m_{\gamma}[6]$. The axion-photon momentum difference becomes $q=\left(m_{\mathrm{a}}^{2}-m_{\gamma}^{2}\right) / 2 E$ so that for $m_{\mathrm{a}}$ values in the neighborhood of the chosen $m_{\gamma}$ the maximum sensitivity is restored. Varying the gas density allows one to scan an entire range of $m_{\mathrm{a}}$ values, of course at the price of having to take data separately at each density setting. Such a programme was first carried out at the Tokyo 
axion helioscope [9] with recent results [10]. In 2005 CAST transitioned to its second phase (CAST-II), operating with a buffer gas to increase sensitivity to higher axion masses. In the first part of CAST-II from late 2005 to early 2007, data were taken with ${ }^{4} \mathrm{He}$ as a buffer gas, extending the sensitivity to $m_{\mathrm{a}} \lesssim 0.4 \mathrm{eV}$. In this manuscript, we report the results of these measurements that supersede all previous laboratory limits on the axion-photon coupling strength in this mass range.

In general the dispersion relation of photons in matter is a complicated function of energy. The refractive index can be either larger or smaller than unity so that in the medium, the dispersion relation can be either space-like $\left(E^{2}-p^{2}<0\right)$ or time-like $\left(E^{2}-p^{2}>0\right)$. In the former case, e.g. for visible light in air, the mismatch between the axion and photon dispersion relations would be exacerbated. We stress this point because it is sometimes overlooked in the literature. If the photon energy is far above all resonances of the medium it is guaranteed, however, that the dispersion relation is not only time-like, but also that its energy dependence is such as if the photons had an effective mass: $E^{2}-p^{2}=m_{\gamma}^{2}$ where $m_{\gamma}^{2}=\omega_{\text {plas }}^{2}=4 \pi \alpha n_{e} / m_{e}$ with $n_{e}$ the electron density, $m_{e}$ the electron mass, and $\alpha=1 / 137$ the fine-structure constant. In other words, for high-energy photons any medium has the same effect as a nonrelativistic plasma with the same electron density. For x-ray energies of a few keV relevant for CAST, one needs to use a low- $Z$ gas such as hydrogen or helium [6]. In the first part of CAST-II we use ${ }^{4} \mathrm{He}$, allowing us to go up to a pressure of $16.4 \mathrm{mbar}$, the ${ }^{4} \mathrm{He}$ vapor pressure at the operating temperature of $1.8 \mathrm{~K}$ of the superconducting magnet, corresponding to $m_{\gamma} \approx 0.4 \mathrm{eV}$. To reach larger masses up to about $1.2 \mathrm{eV}$ we use ${ }^{3} \mathrm{He}$ in the second part of CAST-II.

CAST and previous helioscope experiments are sensitive to axion-like particles within a certain region in the two-parameter plane spanned by $g_{\mathrm{a} \gamma}$ and $m_{\mathrm{a}}$. The main motivation, however, is to search for QCD axions that appear as a consequence of the Peccei-Quinn mechanism to solve the CP problem of strong interactions [14]. For these particles we have

$$
\begin{aligned}
& g_{\mathrm{a} \gamma}=\frac{\alpha}{2 \pi} \frac{1}{f_{\mathrm{a}}}\left(\frac{E}{N}-\frac{2}{3} \frac{4+z}{1+z}\right), \\
& m_{\mathrm{a}}=\frac{z^{1 / 2}}{1+z} \frac{f_{\pi} m_{\pi}}{f_{\mathrm{a}}},
\end{aligned}
$$

where $f_{\mathrm{a}}$ is the Peccei-Quinn scale or axion decay constant, a free parameter of the theory, $m_{\pi}$ is the pion mass, $f_{\pi}=92 \mathrm{MeV}$ the pion decay constant, $E / N$ the ratio of the electromagnetic and colour anomalies of the axial current associated with the PecceiQuinn $U(1)$ symmetry, and $z=m_{\mathrm{u}} / m_{\mathrm{d}}$ the up/down quark mass ratio. Therefore, in the $g_{\mathrm{a} \gamma}-m_{\mathrm{a}}$ plane, the locus for QCD axions is given by

$$
g_{\mathrm{a} \gamma}=\frac{\alpha}{2 \pi}\left(\frac{E}{N}-\frac{2}{3} \frac{4+z}{1+z}\right) \frac{1+z}{z^{1 / 2}} \frac{m_{\mathrm{a}}}{m_{\pi} f_{\pi}},
$$

sometimes called "the axion line." Since $E / N$ is a model-dependent parameter of order unity and since $z$ is somewhat uncertain [15], QCD axions, if they exist, presumably 
live somewhere within a relatively narrow band in the $g_{\mathrm{a} \gamma}-m_{\mathrm{a}}$ plane shown as a yellow band in figure 7. While the CAST-I limits did not yet intersect the axion band, the CAST-II results reported below begin to encroach into it.

The CAST magnet, the detectors, and the Sun as an axion source were described in detail in the final CAST-I paper [12] and [22-24]. In section 2 we describe the modifications of the CAST experimental set-up necessary to inject a buffer gas in the magnet pipes with the required precision, followed by a description of the strategy that we have followed for the data taking. We describe our data analysis and provide new limits in section 3 before concluding in section 4 .

\section{Upgrade and strategy of the CAST experiment for Phase II}

The setup of the CAST experiment has been described elsewhere $[11,16]$. Here we focus on the upgrade that was done to allow operation with ${ }^{4} \mathrm{He}$ buffer gas in the cold magnet bore. This upgrade was the first part of a transition to a more complex system designed for eventual operation at higher buffer gas densities using ${ }^{3} \mathrm{He}$. The system was designed after a series of measurements and tests during resistive transitions (quenches) of the superconducting magnet in June 2005. It was found that that the helium pressure in the cold bore rose by a factor 13 in the first 3 seconds after the quench trigger, and by a factor 20 in 120 seconds, when the gas in the cold bore tubes was not extracted.

During the ${ }^{4} \mathrm{He}$ run the density of the helium in the cold bores was increased in daily steps equivalent to a pressure step of 0.08 mbar at $1.8 \mathrm{~K}$ in the cold bores and in the pipework linking it to the gas system outside. In order to be able to reproduce the density settings precisely, the steps were controlled by injecting a precisely metered quantity of ${ }^{4} \mathrm{He}$ gas into the cold bores. The metering was based on a precise volume with temperature control within $0.1^{\circ} \mathrm{C}$, and on a metrology grade pressure gauge. The density step was determined so as to shift the peak in the axion mass acceptance by one FWHM that provides a sizeable overlap with the previous setting. Thus by making a

series of steps, resulting in a rather smooth scan of the axion mass range, the discovery potential is maximized and has no substantial mass gaps. The system was designed to operate up to 16.4 mbar pressure at $1.8 \mathrm{~K}$, with a stable and homogeneous density along the cold bores, and with an accuracy and reproducibility of equivalent pressure settings better than 0.01 mbar and 0.1 mbar, respectively [17-19]. The homogeneity of the density along the cold bores of the magnet is ensured by the efficient thermal coupling with the subcooled superfluid liquid helium bath filling the magnet vessel. Moreover, as the buffer gas is sealed within the volume including the cold bores and the dead volumes of the linking pipework, the density remains sufficiently constant despite of the small, uncorrelated fluctuations of the temperatures of the magnet and of the dead volumes, because care was taken to minimise the volume of the external pipework connected to the cold bore. During the initial tests spontaneous thermo-acoustic oscillations (TAOs) were observed in the pipework formed by the cold bores and their connections up to the room-temperature shut-off valves. The standing wave has $3.7 \mathrm{~Hz}$ fundamental frequency 
in our system and is driven by non-linear forces in the pipes with large temperature gradient. As a consequence the density varies in time and space in the cold bores, which results in a loss of sensitivity and control of the axion peak width. In order to avoid this effect, damping elements were installed inside the interconnecting pipework to entirely eliminate the conditions that generate the TAO. The tests during magnet quenches were required also for the design of the ${ }^{3} \mathrm{He}$ gas system components and of the passive and active safety measures to protect the cold X-ray windows against the pressure surges due to the quenches. This was not used during the operation with ${ }^{4} \mathrm{He}$ as buffer gas reported here, and will be the subject of a forthcoming paper.

The effect of the gravity in the gas density in the cold bores was also studied and was found to be negligible even if the cold bore tubes were operated vertically. The smallness of the maximum tilt angle of $\pm 8^{\circ}$ reduces the effect further.

A key element in the system are the x-ray windows that confine the buffer gas axially. Four windows were installed at the entrance and exit of the two cold bores of the magnet. These windows must have a high x-ray transmission in the range of $2 \mathrm{keV}$ to $8 \mathrm{keV}$. This requires the use of very thin low Z-material, such as beryllium or plastic. Additional requirements for these windows are: i)resistance to static and dynamic pressures during normal operation and when the magnet quenches, ii)low permeability to helium and iii)transparency at visible wavelengths for both visual inspection and for laser alignment of the x-ray telescope and CCD relative to the axis of the cold bore.

In order to fulfill these requirements, we have made the windows of $15 \mu \mathrm{m}$ thick polypropylene film, supported by an electro-eroded grid (strongback) structure to withstand the 150 mbar pressure difference of future normal operation with ${ }^{3} \mathrm{He}$, as well as the rapid pressure rise that may happen during a quench of the magnet. The strongback obscures $12.6 \%$ of the geometric area. Although polypropylene has a large helium permeation rate at room temperature, this rate is reduced by at least 4 orders of magnitude at cryogenic temperatures. All windows were pressure tested at 1.5 bar at cryogenic temperatures, and the results of leak tests showed values better than $1 \times 10^{-7} \mathrm{mbarLs}^{-1}$ at $60 \mathrm{~K}$. The design and cryogenic test results of the windows have been described briefly in [17].

The pressure rise due to a magnet quench, at the maximum density attained during the runs with ${ }^{4} \mathrm{He}$ buffer gas, was limited to about 300 mbar. During the future runs with ${ }^{3} \mathrm{He}$, a quench in a closed system would result in a maximum pressure of $2.7 \mathrm{bar}$, which requires the implementation of a safety system to limit the pressure to about 1 bar, as will be reported in a forthcoming paper.

The transmission of the foil glued to the strongback was measured at the PANTER x-ray facility[20]. The results of the measurements compared to a calculated transmission using NIST data and including the effect of the strongback are shown in figure 1. The mean calculated and measured transmission are in excellent agreement, with an overall difference of about 1\%. The absorption edge of the polypropylene foil defined the low-energy x-ray cut-off for low densities of the gas; however at increasingly higher densities the absorption of the gas dominates, whereas at low densities the 

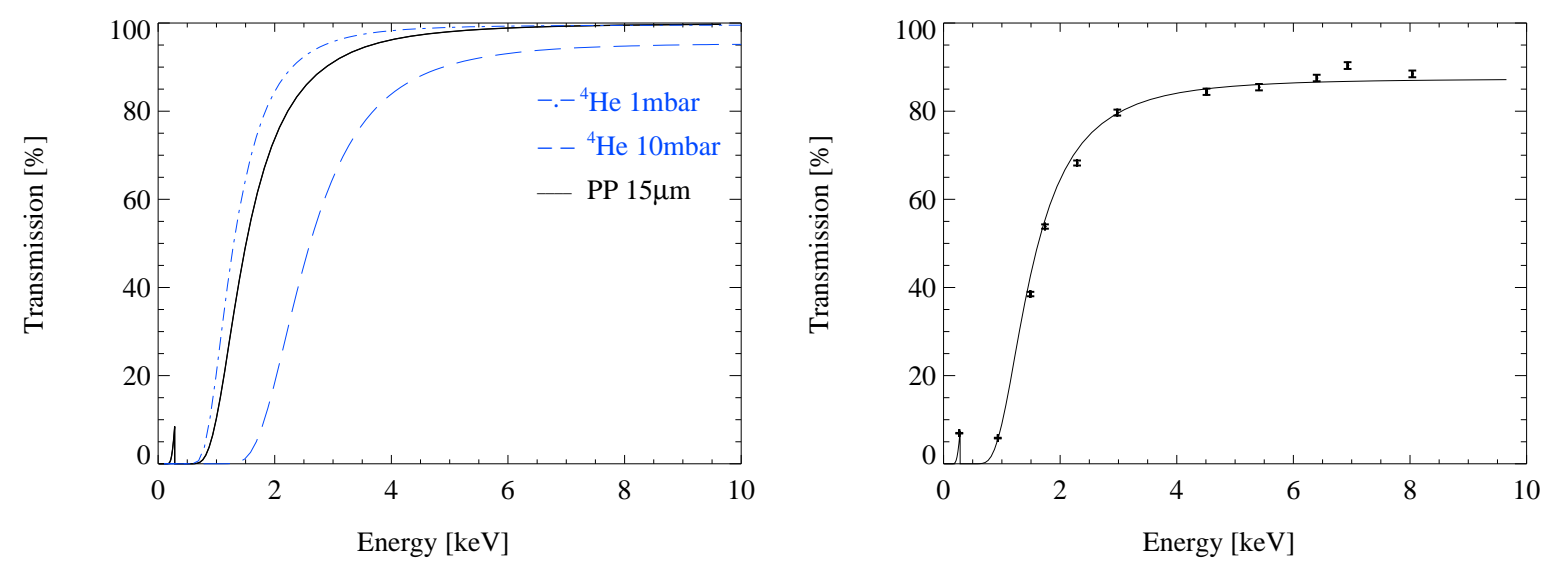

Figure 1. Left:calculated transmission of a $15 \mu \mathrm{m}$ film of polypropylene compared with that of $10 \mathrm{~m}$ of ${ }^{4} \mathrm{He}$ gas at $1.8 \mathrm{~K}$ and at two different pressures. Right: transmission of a $15 \mu \mathrm{m}$ thick polypropylene film glued to a stainless steel strongback. The black points show the measurements performed at the PANTER x-ray facility, with errors smaller than the points. The black curve shows the calculated transmission using the NIST data and taking into account the $12.6 \%$ transmission loss due to the strongback.

transmission of the polypropylene dominates as shown in the left plot of figure 1.

The ${ }^{4} \mathrm{He}$ gas system was operational from November 2005 when the first ${ }^{4} \mathrm{He}$ runs took place. In 2006 the data-taking period lasted for 9 months. The density was changed in daily steps equivalent to 0.08 mbar at $1.8 \mathrm{~K}$, covering equivalent pressures up to 13.4 mbar at $1.8 \mathrm{~K}$. This allowed CAST to scan a new axion mass range between 0.02 and $0.39 \mathrm{eV}$. During 2007, following a technical design review [19], a more sophisticated and complex gas system was installed and commissioned which operates with ${ }^{3} \mathrm{He}$ buffer gas, and includes a pressure-limiting safety system and an improved monitoring and control system that will enable CAST to eventually reach an axion mass of about 1.2 $\mathrm{eV}$. This new system will be described in a forthcoming paper.

\subsection{Off-resonance solar axion identification technique}

In the presence of a buffer gas which provides an effective photon mass $m_{\gamma}$, the axionphoton conversion probability given in equation (2) takes the form

$$
P_{\mathrm{a} \rightarrow \gamma}=\left(\frac{g_{\mathrm{a} \gamma} B}{2}\right)^{2} \frac{1}{q^{2}+\Gamma^{2} / 4}\left[1+e^{-\Gamma L}-2 e^{-\Gamma L / 2} \cos (q L)\right],
$$

where $\Gamma$ is the inverse absorption length for photons in a gas and $q=\left(m_{\mathrm{a}}{ }^{2}-m_{\gamma}^{2}\right) /(2 E)$ is the axion-photon momentum difference. In the particular case of vacuum (when $\Gamma \rightarrow 0$ ), equation (6) becomes equation (2).

The coherent axion-photon conversion will occur for $q L \lesssim \pi$, which implies that the CAST experiment will be sensitive only to axion masses in the range

$$
\sqrt{m_{\gamma}^{2}-\frac{2 \pi E}{L}} \lesssim m_{\mathrm{a}} \lesssim \sqrt{m_{\gamma}^{2}+\frac{2 \pi E}{L}} .
$$


For $q L \gtrsim \pi$, the axion-photon momentum mismatch will reduce the sensitivity. During CAST-I, with vacuum inside the magnet pipes, the coherence condition restricted the CAST sensitivity to $m_{\mathrm{a}} \lesssim 0.02 \mathrm{eV}$. In CAST-II, with helium inside the magnet pipes, the coherence can be restored for a very narrow mass range $\left(\Delta m / m \sim 10^{-2}-10^{-3}\right)$ around $m_{\gamma} \approx m_{\mathrm{a}}$ (for $P=6.08 \mathrm{mbar}$, for example, $\Delta m / m$ is 0.008). The axion-photon conversion probability for two cases is shown in figure 2 . In order to cover the whole accessible axion mass range uniformly, the gas pressure has to be varied in appropriate steps. Figure 3 shows the axion-photon conversion probability for three consecutive pressure settings as well as the sum of the three probabilities. During the ${ }^{4} \mathrm{He}$ phase, the axion mass range $0.02 \mathrm{eV}<m_{\mathrm{a}}<0.39 \mathrm{eV}$ was covered with 160 density settings.

The solar axion flux based on the solar model in [21] is very well approximated by (energies in keV)

$$
\frac{d \Phi_{\mathrm{a}}}{d E}=6.02 \times 10^{10} g_{10}^{2} E^{2.481} e^{-E / 1.205} \mathrm{~cm}^{-2} \mathrm{~s}^{-1} \mathrm{keV}^{-1},
$$

while the differential flux of photons expected at the end of the magnet in case of the coherent conversion is given by

$$
\begin{aligned}
\frac{d \Phi_{\gamma}}{d E} & =\frac{d \Phi_{\mathrm{a}}}{d E} P_{\mathrm{a} \rightarrow \gamma} \\
& =0.088 g_{10}^{4}\left(\frac{L}{9.26 \mathrm{~m}}\right)^{2}\left(\frac{B}{9.0 \mathrm{~T}}\right)^{2} E^{2.481} e^{-E / 1.205} \mathrm{day}^{-1} \mathrm{~cm}^{-2} \mathrm{keV}^{-1},
\end{aligned}
$$

where $g_{10}=g_{\mathrm{a} \gamma} /\left(10^{-10} \mathrm{GeV}^{-1}\right)$. CAST developed a novel technique for solar axion identification using the off-resonance spectral distribution. The expected photon spectrum depends on the resonance mismatch between $m_{\mathrm{a}}$ and $m_{\gamma}$ as shown in figure 4 . The characteristic spectral distribution can be used for additional confirmation of the signal as a solar axion. We remark that this possibility, to the best of our knowledge, was never used before in axion search experiments.

\section{Data analysis}

During the data-taking period with ${ }^{4} \mathrm{He}$ in the magnet bores in 2005 and 2006, the x-ray detectors were operated in the same configuration as for the 2004 data taking period [12,22-24], except for minor improvements. The time projection chamber (TPC) [23], covering both bores of the east end of the magnet looking for axion-tophoton conversion during sunset, had reduced electronic noise, using improved low voltage power supplies. At the other end, looking for x-rays from axion-to-photon conversion during sunrise, two detection systems were installed: a gaseous micromegas chamber (MM) [24] and an x-ray telescope consisting of x-ray optics coupled to a pnCharge Coupled Device (CCD) [22] as a focal plane detector. The use of the x-ray mirror system suppresses the background by a factor of about 155 since the potential signal from the magnet acceptance area of $14.5 \mathrm{~cm}^{2}$ is focused to a spot of roughly $9.3 \mathrm{~mm}^{2}$ on the CCD chip, thus improving the signal to background ratio by the same factor. The vacuum system of the telescope and the CCD was upgraded as well as the 


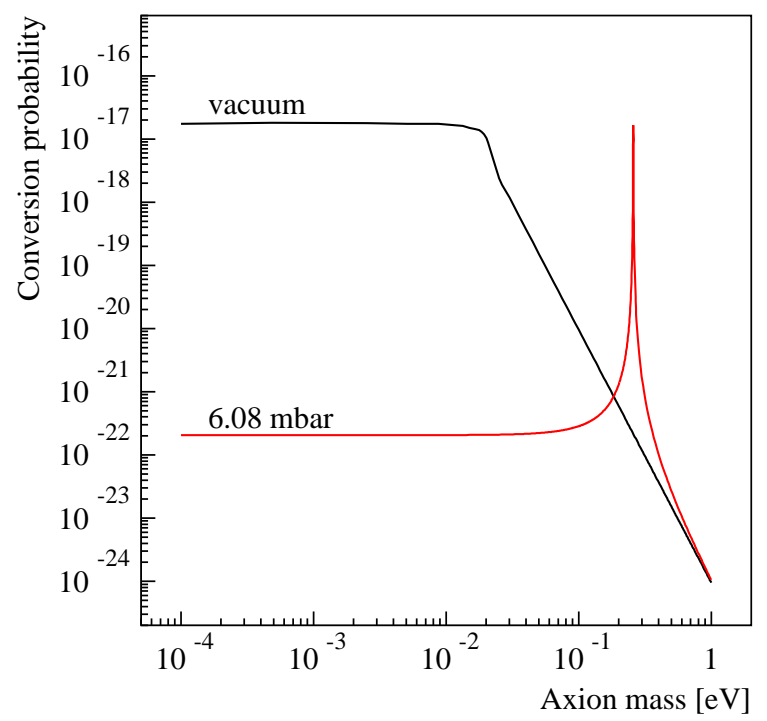

Figure 2. Axion-photon conversion probability versus axion mass. The black line corresponds to vacuum inside the magnet pipes and the red line to one particular helium density setting. Axion-photon coupling constant of $1 \times 10^{-10} \mathrm{GeV}^{-1}$ is assumed.
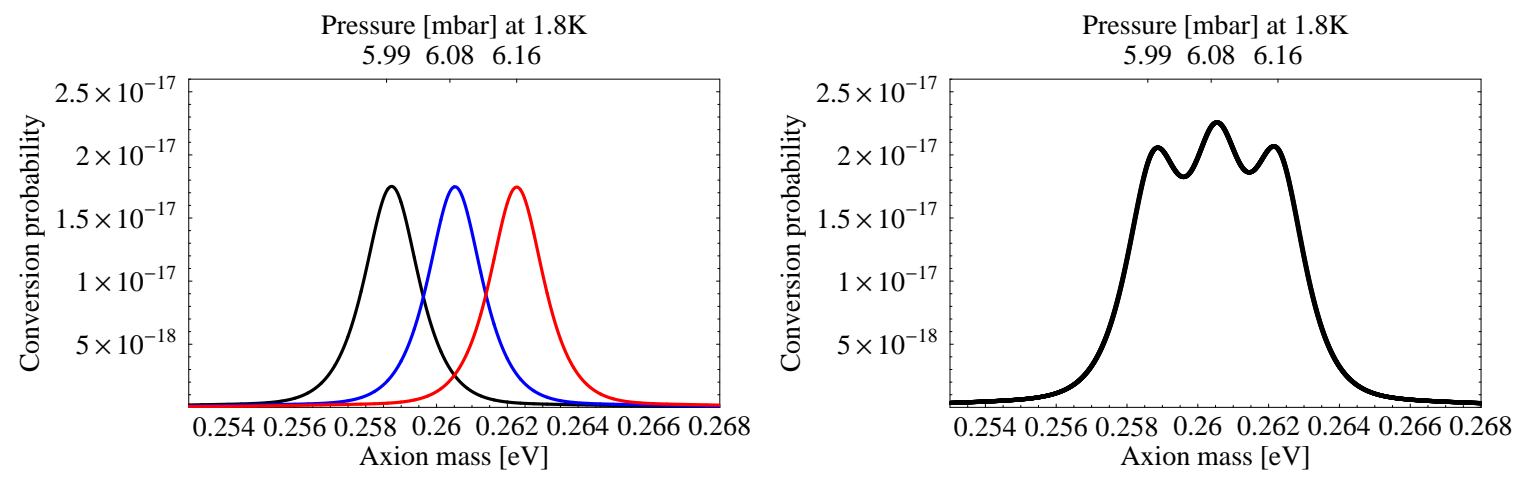

Figure 3. Axion-photon conversion probability versus axion mass for three consecutive density settings on the left. On the right the same plot for the sum of the three probabilities is shown. An axion-photon coupling constant of $1 \times 10^{-10} \mathrm{GeV}^{-1}$ is assumed.

control software resulting in a safer and more flexible mode of operation. A new MM detector was installed for Phase II which had an improved performance with respect to the one used previously: a reduction of the copper fluorescence due to detector materials, which used to dominate the detector background, was achieved by the introduction of a gold-coated amplification mesh.

As during CAST-I, axion-sensitive data were taken when the magnet was pointing to the sun (about $2 \times 1.5 \mathrm{~h}$ per day), while the rest of the time was used to measure background continuously only interrupted by daily calibrations. The density setting was changed once a day so that both sunrise and sunset detectors could cover all settings. 

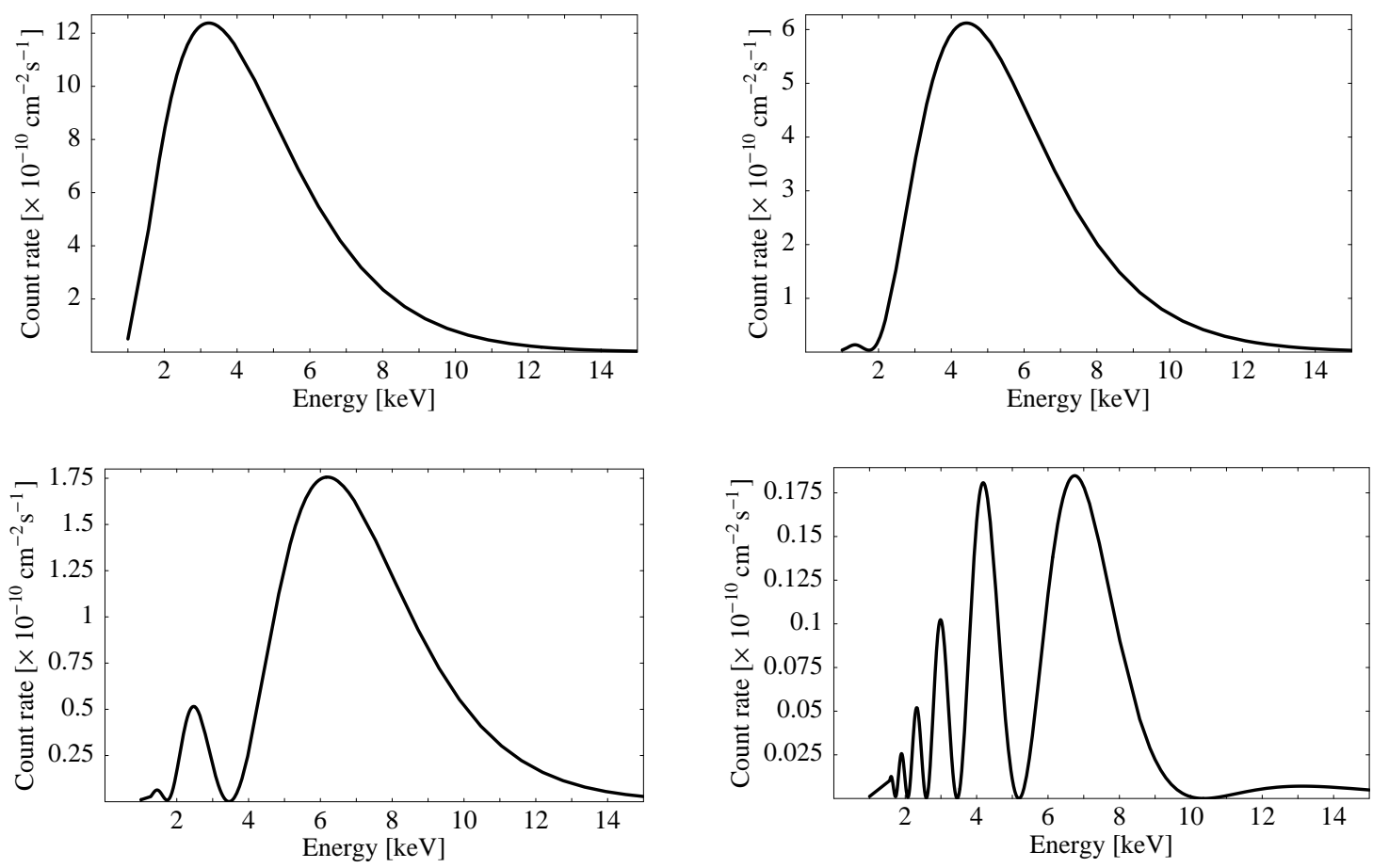

Figure 4. Expected photon spectra depending on the shift $S=m_{\gamma}-m_{a}$ from the resonance: $S=0$ (top left), $S=\mathrm{FWHM} / 2$ (top right), $S=$ FWHM (bottom left) $S=3 \times$ FWHM (bottom right). Axion-photon coupling constant of $1 \times 10^{-10} \mathrm{GeV}^{-1}$ is assumed.
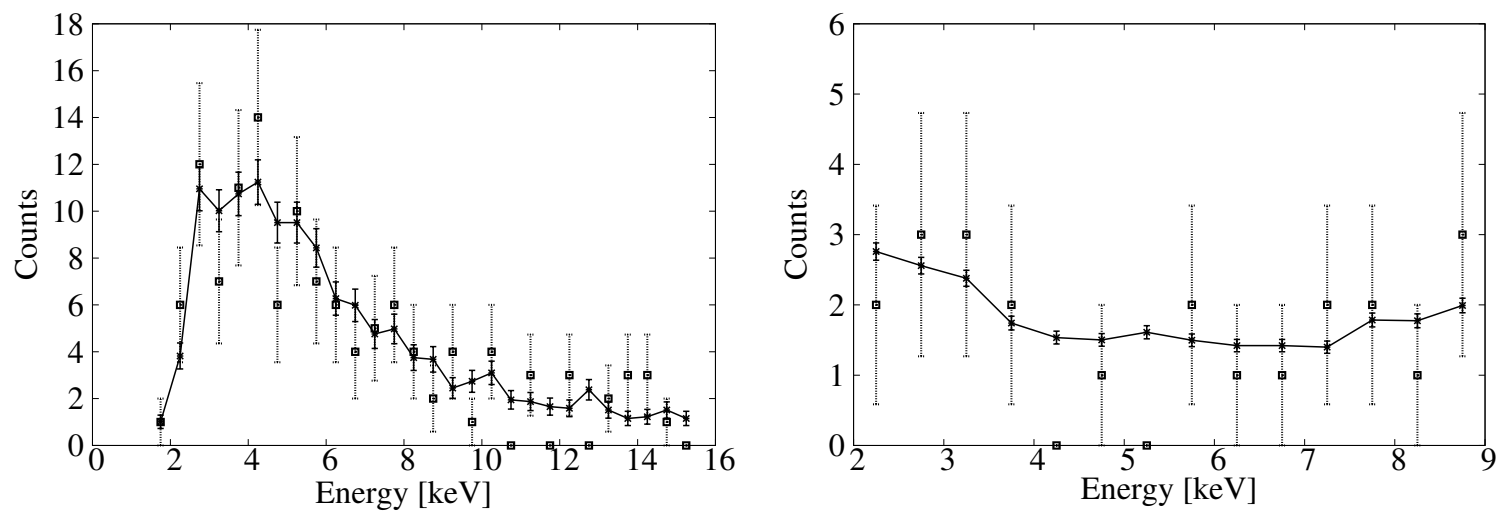

Figure 5. Energy distribution of events recorded during the tracking run (stars with dashed line) at pressure setting $P_{k}=8.909$ mbar compared to background data (empty squares with continuous line) for the TPC (left) and the Micromegas (right) detectors respectively.

Small shutdown periods, for a single detector, due to replacements of components, maintenance or upgrades did occur. As a general rule, the pressure step was repeated if more than one of the detectors were off.

An example of the energy distribution of the events during the tracking run at the density setting $P_{k}=8.909$ mbar at $1.8 \mathrm{~K}$ compared to background data are shown in 

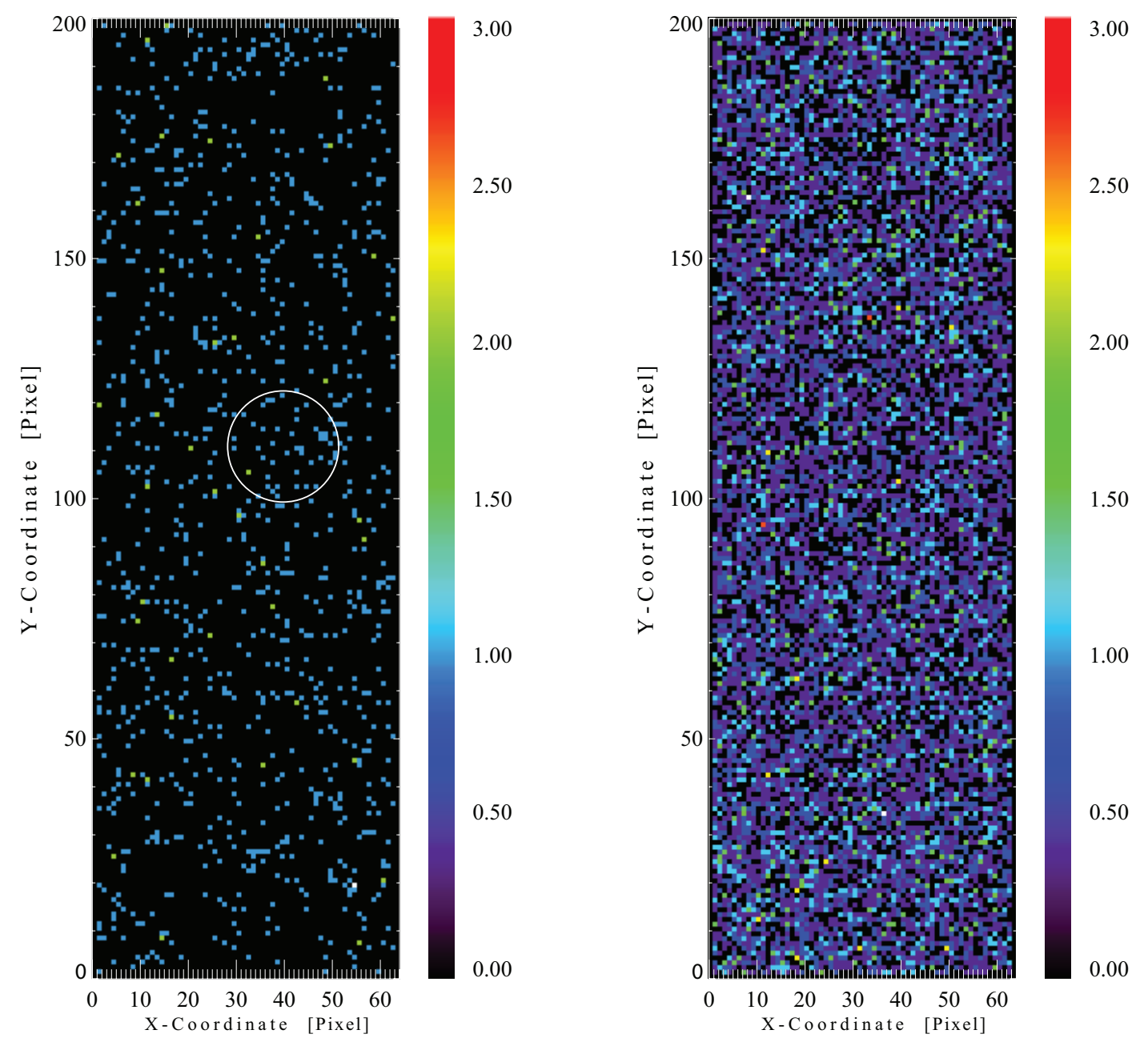

Figure 6. Left: Spatial distribution of events observed during sun tracking by the CAST x-ray telescope during the 2005/2006 data taking period (189 solar observations). The intensity is given in counts per pixel and is integrated over the tracking period of $294.8 \mathrm{~h}$ accounting to 45 counts between 1 and $7 \mathrm{keV}$. The white circle represents the expected size and position of the potential signal, which would be an image of the sun's hot inner axion-producing region. Right: Background spatial distribution as observed by the CAST x-ray telescope during the 2005/2006 datataking period. The intensity is given in counts per pixel and integrated over the full observation period of $2741.5 \mathrm{~h}$.

figure 5 for the TPC and MM detectors. The background data shown in the plots is the effective background used to calculate the axion-photon coupling constant. Figure 6 shows the background data observed in the CCD detector (area $3 \times 1 \mathrm{~cm}^{2}$ ) during the ${ }^{4} \mathrm{He}$ run. The solar axion signal is expected to cover a circular spot of $\approx 9.3 \mathrm{~mm}^{2}$ and is indicated in figure 6 by the white circle. The stability of the alignment of the optical axis of the x-ray telescope to the magnet axis has been monitored with an external x-ray source to an accuracy of \pm 0.5 pixel. Therefore, the location of the potential signal spot on the CCD chip is known with the same precision throughout the run. A summary of the data acquired with each of the detectors for both solar tracking and background 
data is given in table 1 . In order to extract the final result the data from the three detectors are combined.

Table 1. Summary of data taken during the ${ }^{4} \mathrm{He}$ phase. Average background rates are given for each detector.

\begin{tabular}{cccccc}
\hline & Steps & $\begin{array}{c}\text { Tracking } \\
(\mathrm{h})\end{array}$ & $\begin{array}{c}\text { Background } \\
(\mathrm{h})\end{array}$ & $\begin{array}{c}\text { Rate } \\
\mathrm{keV}^{-1} \mathrm{~s}^{-1} \mathrm{~cm}^{-2}\end{array}$ & $\begin{array}{c}\text { Energy Range } \\
\mathrm{keV}\end{array}$ \\
\hline CCD & 147 & 294.8 & 2758.1 & $(8.66 \pm 0.06) \times 10^{-5}$ & $1-7$ \\
TPC & 154 & 304.1 & 4346.6 & $(7.68 \pm 0.01) \times 10^{-5}$ & $2-15$ \\
MM & 159 & 336.6 & 3115.0 & $(4.75 \pm 0.02) \times 10^{-5}$ & $2-9$ \\
\hline
\end{tabular}

During Phase I, the presence of a solar axion would have been evident over the entire data taking period of roughly one year.

The signal to be looked for in the CAST-II data would be present only in a few trackings centered at the density which matches the axion mass observation corresponding to one density setting. The effective exposure time at a given axion mass is approximately 100 minutes, during which a very low number of counts is expected as background (e.g. for the x-ray telescope only about 0.26 counts are expected in the spot area during one tracking). Hence, the sensitivity of CAST in phase II is statistics-limited by the number of expected background events.

In order to extract an axion signal or to derive an upper limit on its coupling constant from CAST-II data, one has to take into account the fact that the axion signal $s$ depends on the density at step $k$ at which data are taken, $s=s_{k}\left(g_{\mathrm{a} \gamma}, m_{\mathrm{a}}\right)$, and that $s_{k}$ is maximum when $m_{\mathrm{a}}$ matches the gas-induced photon mass $m_{k}, m_{k}^{2}=4 \pi \alpha n_{e k} / m_{e}$, $n_{e k}$ being the electron density at setting $k$, but quickly drops as $m_{k}$ deviates from $m_{\mathrm{a}}$. A standard likelihood function can be built for a single step $k$ based on the Poissonian probability distribution,

$$
\mathcal{L}_{k}\left(g_{\mathrm{a} \gamma}, m_{\mathrm{a}}\right)=\frac{P_{k}}{P_{0 k}}=\frac{P\left(\left\{n_{i}\right\}_{k} ;\left\{\mu_{i}\right\}_{k}\right)}{P\left(\left\{n_{i}\right\}_{k} ;\left\{n_{i}\right\}_{k}\right)},
$$

where

$$
P\left(\left\{n_{i}\right\}_{k} ;\left\{\mu_{i}\right\}_{k}\right)=\prod_{i} e^{-\mu_{i k}} \frac{\mu_{i k}^{n_{i k}}}{n_{i k} !},
$$

$n_{i k}$ is the observed number of counts in energy bin $i$ and density step $k$, and $\mu_{i k}$ is the expected number of counts in each bin, estimated as the sum of expected background counts plus the axion signal $\mu_{i k}=b_{i k}+s_{i k}\left(g_{\mathrm{a} \gamma}, m_{\mathrm{a}}\right)$. The estimation of $b_{i k}$ is done using experimental data taken in non-tracking conditions, following different prescriptions that are discussed below. The statistical uncertainty in $b_{i k}$ is not taken into account in equation (10) since its effect on the final result has been shown to be negligible by a dedicated Monte-Carlo simulation. 
Data from different density steps can be combined by multiplying the corresponding $\mathcal{L}_{k}$,

$$
\mathcal{L}\left(g_{\mathrm{a} \gamma}, m_{\mathrm{a}}\right)=\prod_{k} \mathcal{L}_{k}\left(g_{\mathrm{a} \gamma}, m_{\mathrm{a}}\right) .
$$

The use of $\mathcal{L}$ to extract statistical information, like best-fit values or confidence intervals, provides a method to account for neighbouring pressure steps and off-resonance axion masses. The product in equation (12) runs in principle over all $k$ settings, in practice, however, only those settings close to the axion mass $m_{\mathrm{a}}$ evaluated contribute to the final likelihood function, a fact used to reduce computation time. In order to combine the data from the three x-ray detectors, the calculated $\mathcal{L}$ in equation (12) for individual detectors are multiplied.

The final step is now similar to the one followed in our previous analysis [12]. A best-fit value $g_{\text {min }}^{4}$ is obtained after maximisation of $\mathcal{L}$ for a fixed value of $m_{\mathrm{a}}$. The results are compatible with absence of signal, and therefore we can express our result as an upper limit on the axion photon coupling with a 95\% confidence level, $g_{95}^{4}$. To do that we follow the Bayesian approach, which consists in considering the Bayesian probability $P\left(g^{4} \mid g_{\text {min }}^{4}\right)$ with a prior distribution uniform in $g^{4}$ and integrating it from zero to $95 \%$ of its area, in order to find $g_{95}^{4}$. This value is computed for many values of the axion mass $m_{\mathrm{a}}$ in order to configure the full exclusion plot shown in figure 7 . This plot shows the combined CAST-I and ${ }^{4} \mathrm{He}$ part of CAST-II results (blue line) along with the constraints from the Tokyo helioscope [8-10] and HB stars [13,25]. The vertical line (HDM) is the Hot Dark Matter limit for hadronic axions $\left(m_{\mathrm{a}}<1.0 \mathrm{eV}\right)[28,30]$ inferred from observations of the cosmological large-scale structure. The yellow band represents typical theoretical models with $|E / N-1.95|$ in the range $0.07-7$ where the green solid line corresponds to the case $E / N=0$. The red dashed line shows our prospects for the ${ }^{3}$ He run started in March 2008. The plot shows an increase of sensitivity at discrete masses (e.g. $m_{\mathrm{a}} \sim 0.2 \mathrm{eV}$ corresponding to a pressure $P_{k}=3.747$ mbar at $1.8 \mathrm{~K}$ ). This reflects the fact that more time was spent at these pressure settings in order to follow what resulted to be statistical fluctuations. A close-up of the same plot showing the axion mass range explored in the ${ }^{4} \mathrm{He}$ part of CAST-II is also shown.

The influence of systematic uncertainties on the best-fit value of $g^{4}$ and on the upper limit of $g_{\mathrm{a} \gamma}$ has been studied for all experimental parameters entering the analysis: magnet length and field, detector efficiencies, window transparencies, etc. Most of these sources of uncertainty have negligible effects on the final result (less than 1\%), the exception being the background definitions and, for the x-ray telescope, the tracking precision.

Any pointing inaccuracy affects negatively the effective area of the telescope and the expected location and size of the signal spot on the CCD. For our stated accuracy of 0.01 degree, these effects have been quantified to introduce a systematical uncertainty in the final limit of less than $3 \%$.

Regarding the background determination, different prescriptions have been used in order to estimate the systematic uncertainty in our ability to measure the background 
of each detector for each density step, induced by possible uncontrolled dependencies of the background on time, position or other experimental conditions.

For the TPC detector, the background $b_{i k}$ to be used for a specific density step $k$ is usually defined using data taken in the non-tracking runs of the same day(s) (i.e. same pressure in the magnet) as the tracking run of that step. Alternatively, data from neighboring days (up to 5 days after and before) have also been used. Another background definition makes use of the tracking data from off-coherence pressure steps. For the MM detector, in order to check for possible diurnal effects, background measurements taken at different daytime have been compared. For the case of the CCD, the data outside the solar spot, taken either during tracking or non-tracking runs have been used alternatively or in combination with the data from the spot area.

The variation of the final result due to the use of these different background prescriptions is usually much smaller than $10 \%$ and only in extreme cases of that order. Therefore we estimate that the overall effect of systematic uncertainties on our final combined upper limit of $g_{\mathrm{a} \gamma}$ is less than $10 \%$.

As can be seen in figure 7, CAST extends its exclusion line from axion masses of $0.02 \mathrm{eV}$ (Phase I) up to masses of $0.39 \mathrm{eV}$. For the ${ }^{4} \mathrm{He}$ phase the limit at a given mass is derived from a few hours of data taking only and correspondingly small event numbers causing large statistical fluctuations of the line contour.

For the first time, limits resulting from direct observation have entered the QCD axion model band in the $\mathrm{eV}$ range, excluding an important part of the parameter space.

\section{Conclusions}

The CAST-I search for solar axions has provided the most restrictive observational limit on the two-photon coupling of axions and axion-like particles (ALPs) for $m_{\mathrm{a}} \lesssim$ $0.02 \mathrm{eV}[12]$. In the first part of CAST-II setup, we have used ${ }^{4} \mathrm{He}$ as a buffer gas to provide x-rays with an effective mass within the magnet bores. Varying the gas density in 160 steps, we have extended the search up to $m_{\mathrm{a}} \lesssim 0.4 \mathrm{eV}$. The absence of a signal above background excludes a new range in the $g_{\mathrm{a} \gamma}-m_{\mathrm{a}}$ plane shown in figure 7 that was not previously explored by direct laboratory experiments. We were able to derive a mean upper limit of $2.17 \times 10^{-10} \mathrm{GeV}^{-1}$ in the range $0.02<m_{\mathrm{a}}<0.39 \mathrm{eV}$.

The CAST search has now entered the realistic model parameter space for QCD axions. In contrast to generic axion-like particles, QCD axions unavoidably interact with nucleons so that one expects them to be efficiently emitted from a hot nuclear medium. Accordingly, the well-known energy-loss argument based on the duration of the observed neutrino burst of SN 1987A provides a limit corresponding roughly to

$m_{\mathrm{a}} \lesssim 10^{-2} \mathrm{eV}$. A concise and recent summary of the status of this bound can be found in Sec. 6 of [26] and refs. therein. The SN 1987A limit is a powerful argument that has been applied to many cases other than axions, but on the other hand it suffers from the very sparse statistics of the SN 1987A neutrino burst as well as possibly large systematic uncertainties from the axion emission rates in dense nuclear matter. For sure a CAST 

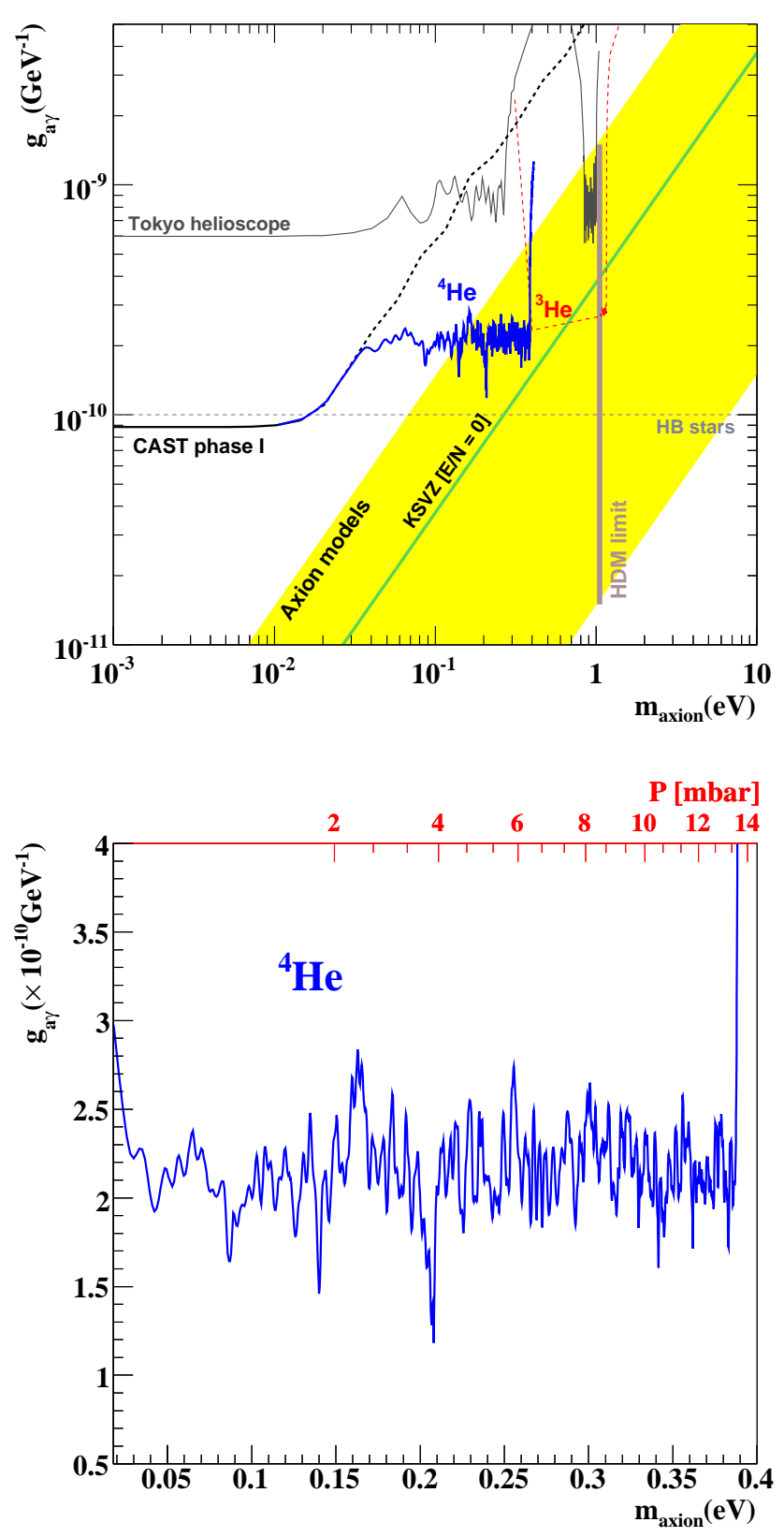

Figure 7. Top: Exclusion plot in the axion-photon coupling versus the axion mass plane. The limit achieved by the CAST experiment (combined result of the CAST-I and ${ }^{4} \mathrm{He}$ part of CAST-II) is compared with constraints from the Tokyo helioscope [810] and HB stars $[13,25])$ discussed in the Introduction. The vertical line (HDM) is the hot dark matter limit for hadronic axions $m_{\mathrm{a}}<1.0 \mathrm{eV} \quad[28,30]$ inferred from observations of the cosmological large-scale structure. The yellow band represents typical theoretical models with $|E / N-1.95|$ in the range $0.07-7$ while the green solid line corresponds to the case when $E / N=0$ is assumed. The red dashed line shows our prospects for the ${ }^{3} \mathrm{He}$ run started in March 2008. Bottom: Expanded view of the limit achieved in the ${ }^{4} \mathrm{He}$ part CAST-II for $m_{\mathrm{a}}$ between $0.02 \mathrm{eV}$ and $0.39 \mathrm{eV}$ corresponding to a pressure scan from 0 to 13.4 mbar. 
detection of axions in the range $m_{\mathrm{a}} \gtrsim 10^{-2} \mathrm{eV}$ would reveal a significant problem with the often-used SN 1987A argument.

In the ongoing second part of CAST-II, we use ${ }^{3} \mathrm{He}$ as a buffer gas, allowing us to reach higher gas pressures at the operating temperature of $1.8 \mathrm{~K}$ and thus to reach axion masses up to about $1 \mathrm{eV}$. In this mass range QCD axions would exist as thermal relic particles in the universe and provide a hot dark matter component similar to neutrinos [27]. Therefore, the usual structure-formation arguments that provide neutrino mass limits can be applied to axions as well [28-31]. Based on the latest cosmological data that are safely in the linear regime of structure formation, a limit on the axion mass of $m_{\mathrm{a}}<1.0 \mathrm{eV}$ at 95\% CL is found [30]. In the long run such cosmological results are likely to improve, with the ultimate goal of detecting the unavoidable neutrino hot dark matter component. If eventually a hot dark matter component above the minimal neutrino contribution is found in cosmological precision data, its interpretation is not necessarily unique and could signify an axion component. In such a case, and if the forthcoming CAST search is unsuccessful, the experimental challenge for axion searches in the sub-eV range is to improve the sensitivity beyond the stellar evolution limits and to cover the full range of plausible axion models that is roughly represented by the yellow band in figure 7 .

\section{Acknowledgments}

CAST wishes to give special thanks to the family Poulin-McGilchrist for kindly granting permission to remove three oak trees which were in line of sight for the sun filming tests carried out each March and September and which are considered an essential crosscheck of the alignment of our experiment. We thank CERN for hosting the experiment and for the contributions of V. Benda, J. P. Bojon, F. Cataneo, R. Campagnolo, G. Cipolla, F. Chiusano, M. Delattre, L. Dufay-Chanat, J.-F. Ecarnot, F. Formenti, D. Fraissard, M. Genet, J. N. Joux, L. Le Mao, A. Lippitsch, L. Musa, R. De Oliveira, A. Onnela, J. Pierlot, S. Prunet, C. Rosset, H. Thiesen, A. Vacca, and B. Vullierme. We acknowledge support from MSES (Croatia) under grant No. 098-0982887-2872, CEA (France), BMBF (Germany) under grant Nos. 05 CC2EEA/9 and 05 CC1RD1/0, the Deutsche Forschungsgemeinschaft (DFG) under grant HO 400/7-1, GSRT (Greece), RFFR (Russia), the Spanish Ministry of Science and Education (MEC) under grants FPA2004-00973 and FPA2007-62833 and the Turkish Atomic Energy Authority. This work was supported in part by the U.S. Department of Energy under Contract No. DEAC52-07NA27344; support from the Laboratory Directed Research and Development Program at LLNL is also warmly acknowledged. We acknowledge helpful discussions within the European Union network on direct dark matter detection of the ILIAS integrating activity (Contract number: RII3-CT-2003-506222).

The CAST collaboration dedicates this paper to the memory of our colleagues E. Arik, F. S. Boydag, O. B. Dogan and I. Hikmet, who perished in the Atlasjet accident in southern Turkey, 30 November 2007. Their friendship, energy and enthusiasm will 
be sorely missed.

\section{References}

[1] Battesti R, Beltran B, Davoudiasl H, Kuster M, Pugnat P, Rabadan R, Ringwald A, Spooner N, Zioutas K, Axion searches in the past, at present, and in the near future, 2008 Lect. Notes Phys. 741, 199 [hep-ex/0705.0615]

[2] Sikivie P, Experimental tests of the invisible axion, 1983 Phys. Rev. Lett. 51, 1415 [Erratum ibid. 52, 695 (1984)]

[3] Dicus D A, Kolb E W, Teplitz V L and Wagoner R V, Astrophysical bounds on the masses of axions and Higgs particles, 1978 Phys. Rev. D 18, 1829

[4] Raffelt G G, Astrophysical axion bounds diminished by screening effects, 1986 Phys. Rev. D 33, 897

[5] Raffelt G and Stodolsky L, Mixing of the photon with low mass particles, 1988 Phys. Rev. D 37, 1237

[6] van Bibber K, McIntyre P M, Morris D E and Raffelt G G, Design for a practical laboratory detector for solar axions, 1989 Phys. Rev. D 39, 2089

[7] Lazarus D M, Smith G C, Cameron R, Melissinos A C, Ruoso G, Semertzidis Y K and Nezrick F A, A search for solar axions, 1992 Phys. Rev. Lett. 69, 2333

[8] Moriyama S, Minowa M, Namba T, Inoue Y, Takasu Y and Yamamoto A, Direct search for solar axions by using strong magnetic field and X-ray detectors, 1998 Phys. Lett. B 434, 147 [arXiv:hep-ex/9805026]

[9] Inoue Y, Namba T, Moriyama S, Minowa M, Takasu Y, Horiuchi T and Yamamoto A, Search for sub-electronvolt solar axions using coherent conversion of axions into photons in magnetic field and gas helium, 2002 Phys. Lett. B 536, 18 [arXiv:astro-ph/0204388]

[10] Inoue Y, Akimoto Y, Ohta R, Mizumoto T, Yamamoto A, Minowa M Search for solar axions with mass around $1 \mathrm{eV}$ using coherent conversion of axions into photons, 2008 Phys. Lett. B 668, 93 [arXiv:astro-ph/0806.2230]

[11] Zioutas K et al. (CAST collaboration), First results from the CERN Axion Solar Telescope, 2005 Phys. Rev. Lett. 94, 121301 [SPIRES] [hep-ex/0411033]

[12] Andriamonje S et al. (CAST collaboration), An improved limit on the axion-photon coupling from the CAST experiment, 2007 JCAP 04, 10 [SPIRES] [hep-ex/0702006]

[13] Raffelt G G, Astrophysical axion bounds, (2008) Lect. Notes Phys. 741, 51 [arXiv:hep$\mathrm{ph} / 0611350]$

[14] Peccei R D, The strong CP problem and axions, 2008 Lect. Notes Phys. 741, 3 [arXiv:hep$\mathrm{ph} / 0607268]$

[15] Yao W M et al. (Particle Data Group), Review of particle physics, 2006 J. Phys. G 33, 1

[16] Zioutas K et al, A decommissioned LHC model magnet as an axion telescope, 1999 Nucl. Instrum. Methods Phys. Res., A $\mathbf{4 2 5} 480$ [astro/ph-9801176]

[17] Niinikoski T O et al., Thin cryogenic X-ray windows, 2008 Submitted to Proc. ICEC 22, Seoul

[18] CAST Collaboration, Status Report of the CAST Collaboration, CERN-SPSC-2005-022

[19] CAST Collaboration, Technical Design Report of the CAST ${ }^{3} H e$ Gas System, SPSC-TDR-001, CERN-SPSC-2006-029

[20] Freyberg M J, Bräuninger H, Burkert W, Hartner G D, Citterio O, Mazzoleni F, Pareschi G, Spiga D, Romaine S and Gorenstein P and Ramsey B D, The MPE X-ray test facility PANTER: Calibration of hard X-ray (15-50 keV) optics, 2005 Experimental Astronomy, 20405

[21] Bahcall J N and Pinsonneault M H, 2004 Phys. Rev. Lett. 92 121301, see also http://www.sns.ias.edu/ jnb/SNdata/sndata.html

[22] Kuster M et al., The X-ray telescope of CAST, 2007 New J. Phys 9, 169 [SPIRES] [hep-ex/0702188]

[23] Autiero D et al., The CAST Time Projection Chamber, 2007 New J. Phys 9, 171 [SPIRES] [hep-ex/0702189] 
[24] Abbon P et al., The Micromegas detector of the CAST experiment, 2007 New J. Phys 9, 170 [SPIRES] [hep-ex/0702190]

[25] Raffelt G G, Stars as Laboratories for Fundamental Physics, 1996 (Chicago, IL: The University of Chicago Press)

[26] Raffelt G G, Astrophysical axion bounds, 2008 Lect. Notes. Phys. 741, 51 [arXiv:hep-ph/0611350]

[27] Moroi T and Murayama H, Axionic hot dark matter in the hadronic axion window, 1998 Phys. Lett. B 440, 69 [arXiv:hep-ph/9804291]

[28] Hannestad S, Mirizzi A and Raffelt G, New cosmological mass limit on thermal relic axions, 2005 JCAP 0507, 002 [arXiv:hep-ph/0504059]

[29] Hannestad S, Mirizzi A, Raffelt G G and Wong Y Y Y, Cosmological constraints on neutrino plus axion hot dark matter, 2007 JCAP 0708, 015 [arXiv:0706.4198 [astro-ph]].

[30] Hannestad S, Mirizzi A, Raffelt G G and Wong Y Y Y, Cosmological constraints on neutrino plus axion hot dark matter: Update after WMAP-5, 2008 JCAP 0804019 [arXiv:0803.1585 [astro-ph]]

[31] Melchiorri A, Mena O and Slosar A, An improved cosmological bound on the thermal axion mass, 2007 Phys. Rev. D 76, 041303 [arXiv:0705.2695 [astro-ph]] 